\title{
TRATAMENTO ARTROSCÓPICO DA OSTEOCONDROMATOSE SINOVIAL NO QUADRIL
}

\author{
ARTHROSCOPIC TREATMENT OF SYNOVIAL \\ OSTEOCHONDROMATOSIS IN THE HIP
}

Giancarlo Cavalli Polesello', Nelson Keiske Ono ${ }^{2}$, Emerson Kiyoshi Honda ${ }^{3}$, Rodrigo Pereira Guimarães ${ }^{4}$, Walter Ricioli Junior ${ }^{5}$, Bruno Gonçalves Schröder e Souza ${ }^{6}$, William Soltau Dani ${ }^{7}$

\section{RESUMO}

Objetivo: O objetivo deste trabalho é mostrar os resultados obtidos no tratamento da osteocondromatose no quadril pela via artroscópica. Métodos: Foram avaliados seis pacientes submetidos à artroscopia do quadril por osteocondromatose sinovial, quanto à função e dor nos períodos pré e pós-operatórios. $\mathrm{O}$ tempo de queixa pré-operatória variou de nove a 48 meses, média de 28,2 meses e o seguimento variou de oito a 25 meses, média de 17,1 meses. A idade média foi de 45,1 anos, quatro pacientes $(66,6 \%)$ eram do sexo feminino e o lado direito foi acometido em cinco casos (83,3\%). Resultados: Quanto aos resultados pelos critérios de Harris modificados por Byrd, a pontuação evoluiu de média de 54,1 para 90,4 pontos e a escala de expressões faciais para quantificação da dor variou de média de 1,7 ponto para 5,1 pontos. Conclusão: A artroscopia é boa alternativa para o tratamento da osteocondromatose no quadril, apesar de ser necessário maior tempo de seguimento para afirmar isso categoricamente. Os resultados observados até então são animadores. Trata-se de procedimento pouco invasivo e que permite boa recuperação.

Descritores - Quadril; Artroscopia; Condromatose sinovial

\begin{abstract}
Objective: The objective of this study is to show the results achieved in the treatment of hip osteochondromatosis by arthroscopy. Methods: Six patients submitted to hip arthroscopy for synovial osteochondromatosis have been assessed for preand postoperative function and pain. The time of preoperative complaint ranged from nine to 48 months, in an average of 28.2 months and the follow-up ranged from eight to 25 months (mean: 17.1 months). Mean age was 45.1 years, with four female patients (66.6\%) and the right side affected in five cases (83.3\%). Results: Concerning the results according to Byrd-modified Harris' criteria, the mean score evolved from 54.1 to 90.4, and the mean score on the face expressions scale for pain assessment ranged from 1.7 to 5.1. Conclusion: arthroscopy is a good alternative for hip osteochondromatosis treatment, although longer follow-up times are required to strictly stating this. The results found so far are encouraging, constituting a little-invasive procedure allowing good recovery.
\end{abstract}

Keywords - Hip; Arthroscopy; Chondromatosis, Synovial

\footnotetext{
1 - Professor Assistente Doutor da FCMSCSP; Assistente do Grupo de Quadril - FCMSCSP.

2 - Professor Assistente Doutor da FCMSCSP; Chefe do Grupo de Quadril - FCMSCSP.

3 - Professor Assistente Doutor da FCMSCSP; Membro Sênior do Grupo de Quadril - FCMSCSP.

4 - Professor Instrutor Mestre da FCMSCSP; Assistente do Grupo de Quadril - FCMSCSP.

5 - Médico Ortopedista; Assistente do Grupo de Quadril - FCMSCSP.

6 - Médico Ortopedista; Aluno da Pós-graduação da FCMSCSP; Ex-Estagiário do Grupo de Quadril e Fellow da Steadman-Hawkins Clinic, Vail (CO), EUA.

7 - Médico Ortopedista da Clinitrauma Lages-SC; Ex-Estagiário do Grupo de Quadril da FCMSCSP.
}

Trabalho realizado no Departamento de Ortopedia e Traumatologia da Faculdade de Ciências Médicas da Santa Casa de São Paulo. Diretor: Prof. Dr. Osmar Avanzi. Correspondência: Al. dos Aicás, 491, apt ${ }^{\circ} 171$ - 04086-001 - São Paulo, SP, Brasil. Tel./fax: (11) 3225-0958. E-mail: giancarlopolesello@hotmail.com 


\section{INTRODUÇÃO}

A condromatose sinovial é uma metaplasia progressiva, crônica, benigna e rara, na qual a cartilagem é formada nas membranas sinoviais das articulações, bainhas dos tendões ou bursas. Tem origem desconhecida e afeta geralmente uma única articulação ${ }^{(1-8)}$. Alguns dos focos metaplásticos podem destacar-se, produzindo corpos livres. Quando esses corpos se calcificam, a condição é chamada de osteocondromatose sinovial ${ }^{(4)}$. Afeta preferencialmente homens entre 30 e 50 anos e existe possibilidade de recidiva após o tratamento ${ }^{(3,5)}$.

O diagnóstico deve ser considerado em qualquer paciente que apresente mais de cinco corpos livres intracapsulares. Apesar da natureza benigna dessa afecção, os múltiplos corpos livres dentro da cápsula podem causar danos às superfícies articulares com erosão óssea e consequente destruição $\operatorname{articular}^{(4)}$.

Ambroise Paré apud Ginaldi ${ }^{(9)}$ foi o primeiro a relatar essa afecção, em 1558. A primeira descrição histomorfológica foi feita por Jaffe ${ }^{(10)} \mathrm{em} \mathrm{1958,} \mathrm{delineando} \mathrm{as} \mathrm{bases}$ para o diagnóstico histológico da osteocondromatose $\mathrm{e}^{(11)}$.

A localização no quadril não é comum, sendo mais frequente no joelho e no cotovelo ${ }^{(2,3,5-8)}$. Quando afeta o quadril, o diagnóstico é normalmente retardado em virtude do quadro insidioso dos sintomas ${ }^{(2)}$.

O objetivo deste trabalho é mostrar os resultados preliminares obtidos no tratamento da osteocondromatose no quadril pela via artroscópica.

\section{MÉTODOS}

Foram avaliados prospectivamente, no Departamento de Ortopedia e Traumatologia da Santa Casa de São Paulo - Pavilhão Fernandinho Simonsen, seis pacientes submetidos à artroscopia do quadril por osteocondromatose sinovial entre julho de 2004 e julho de 2006 . O trabalho foi previamente submetido à Comissão de Ética em Pesquisa e aprovado sob o $\mathrm{n}^{\mathrm{o}} 317 / 08$.

A idade variou de 41 a 52 anos, média de 45,1 anos. Quanto à distribuição por sexo, quatro $(66,6 \%)$ pacientes eram femininos; o lado direito foi acometido em cinco pacientes $(83,3 \%)$. A cor branca prevaleceu em cinco pacientes $(83,3 \%)$. O tempo de queixa pré-operatória variou de nove a 48 meses, média de 28,2 meses.

O seguimento variou de oito a 25 meses, média de 17,1 meses. Nenhum paciente praticava atividade física regularmente.

O diagnóstico foi feito pela história clínica, pelo exame físico e pelos exames de imagem. Grande parte das radiografias pré-operatórias da bacia não demonstra calcificações, o que pode dificultar o diagnóstico (Figuras 1 e 2).

Ao exame físico, todos apresentavam dor à flexão de $90^{\circ}$, principalmente na região anterior do quadril. Dois pacientes $(33,3 \%)$ relatavam dor para subir e descer escadas, e três $(50 \%)$ tinham dor para entrar e sair do carro ou ao levantar-se.

Para avaliação de resultados, utilizamos o escore de Harris modificado por Byrd (HHS) ${ }^{(12)}$ e a escala de expressões faciais para quantificação da dor $(\mathrm{EEF})^{(13)}$.

Também anotamos as fases da doença como descritas por Milgram ${ }^{(14)}$ : estágio 1- doença intrassinovial ativa com nenhuma formação de nódulo; estágio 2 - desenvolvimento de nódulos na membrana sinovial, (Figuras 3 e 4); estágio 3 - formação de corpos livres articulares (Figura 5).

Avaliamos também os achados artroscópicos durante o procedimento e as complicações pós-operatórias.

Todas as artroscopias foram realizadas pelo mesmo cirurgião.
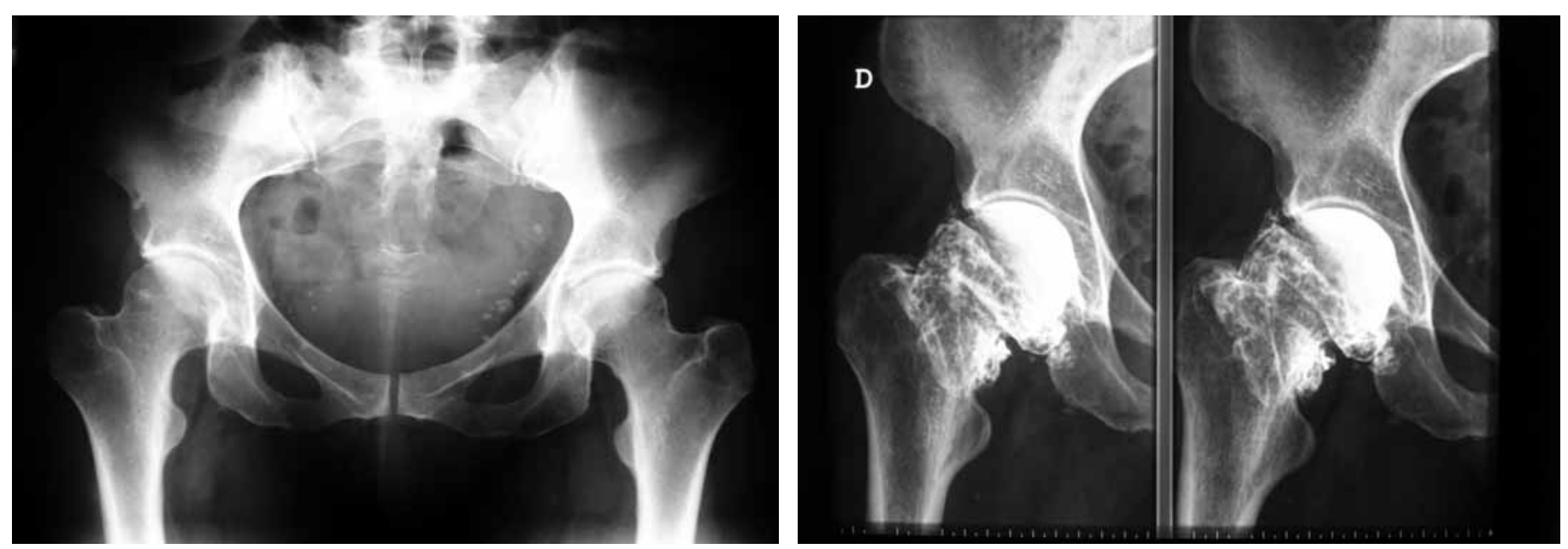

Figuras 1 e 2 - Ausência de sinais radiográficos de corpos livres à radiografia simples, no quadril direito. A artrografia mostrou tratar-se de múltiplos corpos livres intra-articulares, não calcificados 

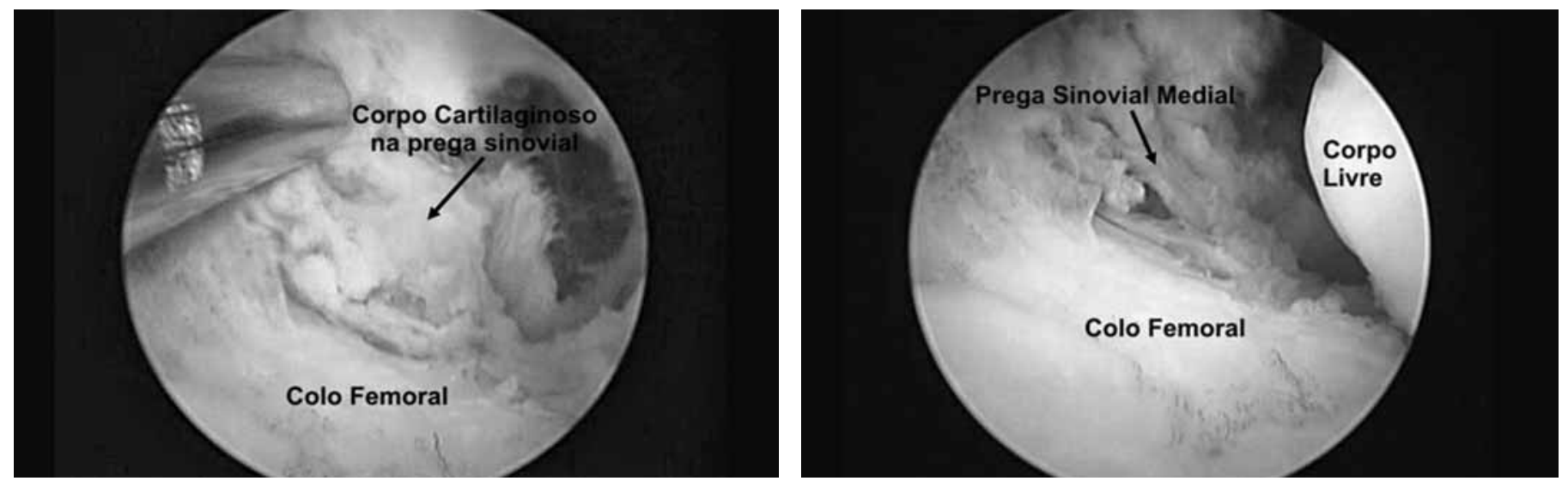

Figuras 3 e 4 - Nódulo cartilaginoso aderido à prega sinovial medial do quadril. Aspecto artroscópico antes e após sua remoção debaixo da prega sinovial

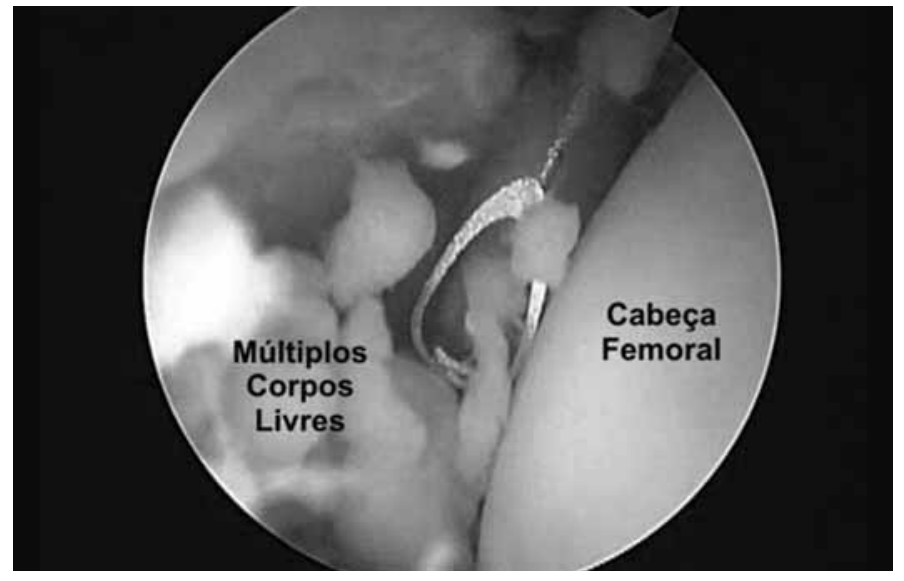

Figura 5 - Múltiplos corpos livres cartilaginosos intra-articulares no quadril

\section{RESULTADOS}

Os pacientes apresentaram melhora clínica e radiográfica; houve melhora do arco de movimento e dos sintomas. Ao exame físico, a dor em flexão e rotação interna melhorou em todos os pacientes. $\mathrm{O}$ retorno às atividades normais foi autorizado oito semanas após a cirurgia.

$\mathrm{O}$ escore de Harris modificado por Byrd ${ }^{(12)}$ variou de 38,5 a 70,4 pontos no pré-operatório (média de 54,1 pontos) e de 79,1 a 95,7 no período pós-operatório (média de 90,4 pontos).

A escala de expressões faciais para quantificação da dor ${ }^{(13)}$ mostrou pontuação pré-operatória que variou de um a três pontos (média de 1,75 ponto) e quatro a seis pontos no pós-operatório (média de 5,1 pontos).

Entre os achados da artroscopia foram evidenciadas lesões da cartilagem articular no acetábulo, acompanhadas de sinovite reacional em três pacientes $(50 \%)$. Observamos neste estudo casos tanto em estágio 2 como 3 , como descritos por Milgram ${ }^{(14)}$, e lesão do lábio aceta- bular em apenas um caso (16,6\%), além de um caso de osteocondroma solitário gigante (Figura 6).

Em todos os casos, o diagnóstico pré-operatório de osteocondromatose sinovial foi confirmado durante o ato cirúrgico e pela biópsia. Não houve complicações ou recidivas até o momento.

\section{DISCUSSÃO}

A osteocondromatose sinovial é considerada uma metaplasia do tecido sinovial ${ }^{(2-8)}$, que afeta a camada subserosa da superfície interna da cápsula articular. É caracterizada pelo desenvolvimento dos corpos cartilaginosos múltiplos, vindos da superfície interna da cápsula ou das outras estruturas formadas por sinóvia, tais como bainhas dos tendões e bursas ${ }^{(1)}$.

A maioria das descrições é de lesões com diâmetro máximo de $3 \mathrm{~cm}$, embora haja raros casos de osteocondromatose solitária gigante, o que observamos nessa série.

Milgram $^{(14)}$ descreveu três fases da doença: estágio 1 - doença intrassinovial ativa com nenhuma formação de nódulo; estágio 2 - desenvolvimento de nódulos na

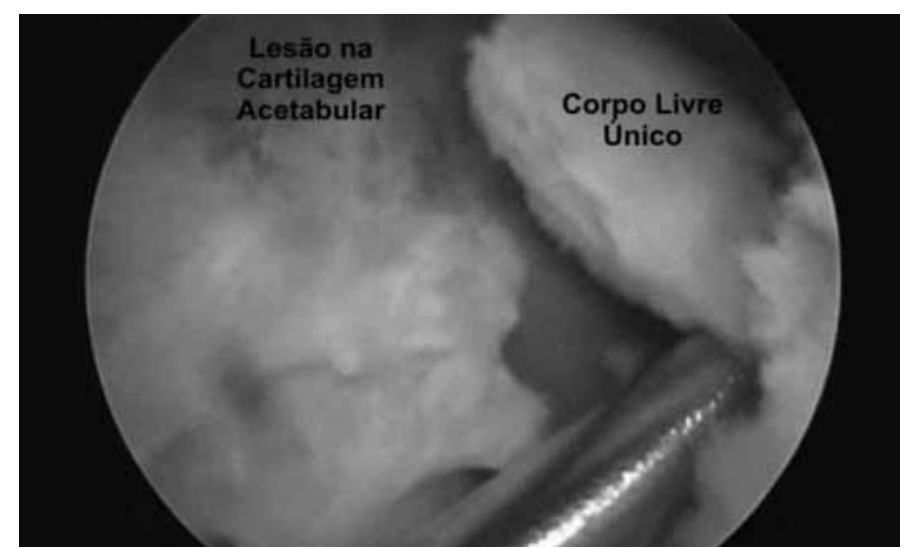

Figura 6 - Presença de corpo livre intracapsular, único com lesão da cartilagem do acetábulo 
membrana sinovial; e estágio 3 - formação de corpos livres articulares. Observamos nas artroscopias os estágios 2 e 3, bem definidos.

A doença ocorre em todas as faixas etárias, mas é predominante na quarta década, sendo duas vezes mais comum em homens ${ }^{(7)}$. Na nossa série tivemos predominância no sexo feminino.

A apresentação clínica é caracterizada geralmente pelos sintomas mecânicos que ocorrem quando os corpos frouxos ou unidos interferem com a função articular, causando limitação do movimento e, eventualmente, bloqueio acompanhado de dor ${ }^{(1,2)}$.

A maioria dos casos ocorre no joelho e cotovelo ${ }^{(2,3,5-8)}$. Existem poucas descrições no quadril ${ }^{(15)}$. Em nosso meio os relatos restringem-se a casos com acometimento do joelho e ombro ${ }^{(16-18)}$.

A característica patológica comum é a extensão das lesões para além da cápsula articular. Localização extraarticular isolada também é documentada, principalmente na bainha dos tendões das mãos e pés. Quando acometem as bursas, provocam dor e rigidez articular, em geral com progressão lenta dos sintomas ${ }^{(7)}$.

As radiografias mostram somente $50 \%$ das lesões, pois a grande parte delas não apresenta calcificação no seu interior, o que também observamos neste estudo ${ }^{(2)}$.

McCarthy e Busconi ${ }^{(19)}$ relatam taxa de $80 \%$ de falsonegativos nos exames de imagem de quadris com dor intratável, incluindo radiografia, artrografia, cintilografia óssea, tomografia computadorizada (TC) e ressonância nuclear magnética (RNM). Em uma revisão de 30 casos, concluíram que os danos condrais eram comuns e às vezes graves, especialmente nas margens das articulações, apesar dos exames normais.

\section{REFERÊNCIAS}

1. Elliott R, Dennien B, Leong A, Van Der Wall H. Synovial osteochondromatosis presenting as a mass in the buttock. Clin Nucl Med. 2001;26(12):1032-3.

2. Krebs VE. The role of hip arthroscopy in the treatment of synovial disorders and loose bodies. Clin Orthop Relat Res. 2003(406):48-59.

3. Lohmann $\mathrm{CH}$, Köster G, Klinger HM, Kunze E. Giant synovial osteochondromatosis of the acromio-clavicular joint in a child. A case report and review of the literature. $\mathrm{J}$ Pediatr Orthop B. 2005;14(2):126-8.

4. Newberg $\mathrm{AH}$, Newman JS. Imaging the painful hip. Clin Orthop Relat Res. 2003;(406):19-28.

5. Campeau NG, Lewis BD. Ultrasound appearance of synovial osteochondromatosis of the shoulder. Mayo Clin Proc. 1998;73(11):1079-81.

6. Marín-Peña O, González-Onandía P, Trell-Lesmes F, Murillo-Vizuete D, Teijeira-Rodríguez A, Galiano JMG. Synovial osteochondromatosis: clinical presentation and treatment. J Bone Joint Surg Br. 2004;86(Suppl 2):139.

7. Birchall D, Khangure MS, Spagnolo DV. Vertebral synovial osteochondromatosis with compressive myelopathy. Spine. 1999;24(9):921-3.

8. Hashimoto N, Okada K. Synovial osteochondromatosis of the retrocalcaneal bursa: a case report. J Bone Joint Surg Am. 1996;78 (11):1741-5

9. Ginaldi S. Computed tomography feature of synovial osteochondromatosis. Skeletal Radiol. 1980;5(4):219-22.

10. Jaffe HL. Tumours and tumourous conditions of the bones and joints. Philadelphia: Lea \& Febijs; 1958.
Alguns autores ${ }^{(1,3,5-7)}$ recomendam a artrotomia seguida de sinovectomia e remoção de todos os corpos livres. Essa conduta não determina o final do processo patológico, mas possibilita melhora da dor e da mobilidade articular. A sinovectomia reduz o potencial do surgimento de novas lesões e retarda a progressão da doença $a^{(1,7)}$. No quadril pode ser necessária a luxação da articulação para obter visibilização adequada com sinovectomia exten$\mathrm{sa}^{(20)}$. Esse tipo de abordagem ampla implica, no entanto, maior morbidade e risco de complicações ${ }^{(20,21)}$.

Num estudo recente, Boyer e Dorfmann ${ }^{(15)}$ relatam a experiência com o tratamento artroscópico da condromatose sinovial do quadril. Resultados bons e excelentes foram obtidos em mais da metade dos pacientes e o seguimento médio foi maior que seis anos, com índice de recidiva em torno de $40 \%$.

Neste trabalho utilizamos a artroscopia para tratar todos os casos, com remoção dos corpos livres e sinovectomia subtotal da articulação. Não tivemos casos de recidiva, embora nosso seguimento seja relativamente curto. Foram relatadas recidivas até 14 anos após tratamento cirúrgico inicialmente bem sucedido ${ }^{(15)}$. A artroscopia do quadril mostrou ser um método alternativo à cirurgia aberta, possibilitando acesso pouco invasivo e com recuperação rápida.

\section{CONCLUSÃO}

Concluímos que a artroscopia é boa alternativa para o tratamento da osteocondromatose sinovial no quadril em nosso meio, apesar de necessitarmos maior tempo de seguimento para afirmar isso categoricamente. Os resultados observados até então são animadores. Tratase de procedimento pouco invasivo e seguro, capaz de permitir boa recuperação.

11. Von Lindern JJ, Theuerkauf I, Niederhagen B, Bergé S, Appel T, Reich RH. Synovial chondromatosis of the temporomandibular joint: clinical, diagnostic, and histomorphologic findings. Oral Surg Oral Med Oral Pathol Oral Radiol Endod. 2002;94(1):31-8.

12. Byrd JW. Avoiding the labrum in hip arthroscopy. Arthroscopy. 2000;16(7):770-3.

13. Pimenta CAM, Teixeira MJ. Avaliação da dor. Rev Med (São Paulo). 1997;76(1):27-35.

14. Milgram JW. Synovial osteochondromatosis: a histopathological study of thirty cases. J Bone Joint Surg Am. 1997;59(6):792-801.

15. Boyer T, Dorfmann H. Arthroscopy in primary synovial chondromatosis of the hip: description and outcome of treatment. J Bone Joint Surg Br. 2008;90(3):314-8.

16. Ramos MRF, Ramos RRM, Santos LA. Condromatose sinovial. Relato de caso. Rev Bras Ortop. 1997;32(9):749-50.

17. Brasil Filho R, Filardi Filho CS, Menitti EI, Baptista MV, Daher SS. Condromatose sinovial: relato de um caso. Rev Bras Ortop. 1997;32(11):921-3.

18. Paim AE, Ferreira DC, Paim A, Almeida RM. Tratamento artroscópico da condromatose sinovial do ombro: relato de caso. Rev Bras Ortop. 2008;43(4):146-9.

19. McCarthy JC, Busconi B. The role of hip arthroscopy in the diagnosis and treatment of hip disease. Orthopedics 1995;18(8):753-6.

20. Lim SJ, Chung HW, Choi YL, Moon YW, Seo JG, Park YS. Operative treatment of primary synovial osteochondromatosis of the hip. J Bone Joint Surg Am. 2006;88(11):2456-64

21. Lim SJ, Park YS. Operative treatment of primary synovial osteochondromatosis of the hip. Surgical Technique. J Bone Joint Surg Am. 2007;89(Suppl 2 Pt.2):232-45. 